\title{
Predictive Nomogram of Subsequent Liver Metastasis After Mastectomy or Breast-Conserving Surgery in Patients With Nonmetastatic Breast Cancer
}

\author{
Anli Yang $\left.{ }^{(}\right)$, Weikai Xiao', Shaoquan Zheng', Yanan Kong', Yutian Zou', \\ Mingyue $\mathrm{Li}^{3}$, Feng $\mathrm{Ye}^{\prime}$, and Xiaoming Xie'
}

\begin{abstract}
Background: Metastasis accounts for the majority of deaths in patients with breast cancer. Liver metastasis is reported common for breast cancer patients. The purpose of this study was to construct a nomogram to predict the likelihood of subsequent liver metastasis in patients with nonmetastatic breast cancer, thus high-risk patient populations can be prevented and monitored.

Methods: A total of 1840 patients with stage I-III breast cancer were retrospectively included and analyzed. A nomogram was constructed to predict liver metastasis based on multivariate logistic regression analysis. SEER database was used for external validation. C-index, calibration curve and decision curve analysis were used to evaluate the predictive performance of the model.

Results: The nomogram included 3 variables related to liver metastasis: HER2 status (odds ratio (OR) I.86, 95\%Cl I.02 to 3.4I; $P=0.045$ ), tumor size (OR 3.62, I.9I to 6.87; $P<0.00 \mathrm{I}$ ) and lymph node metastasis (OR 2.26, I.I8 to 4.34; $P=0.014$ ). The $\mathrm{C}$ index of the training cohort, internal validation cohort and external validation cohort were $0.699,0.8 \mathrm{I} 4$ and $0.79 \mathrm{I}$, respectively. The nomogram was well-calibrated, with no statistical difference between the predicted and the observed probabilities.

Conclusion: We have developed and validated a robust tool enabled to predict subsequent liver metastasis in patients with nonmetastatic breast cancer. Distinguishing a population of patients at high risk of liver metastasis will facilitate preventive treatment or monitoring of liver metastasis.
\end{abstract}

\section{Keywords}

breast cancer, postoperative liver metastasis, nomogram, prediction, validation

Received December 30, 2020. Received revised December 30, 2020. Accepted for publication January 29, 2021.

\section{Introduction}

Breast cancer is one of the leading causes of cancer-related deaths among women worldwide. ${ }^{1}$ Although only $6 \%-10 \%$ of breast cancer patients are diagnosed with metastatic disease, approximately $30 \%$ of women diagnosed with the nonmetastatic disease will relapse after treatment. ${ }^{2,3}$ Breast cancer mainly metastasizes to bone, lung, liver and brain through circulation; among them, the liver is the third most common distant metastatic site of breast cancer. ${ }^{4}$ Liver metastasis is reported to be responsible for approximately $20 \%$ to $35 \%$ of metastatic breast cancer patients' death. ${ }^{5-7}$ Studies have shown that breast cancer patients with liver metastasis exhibit poor

\footnotetext{
' Department of Breast Oncology, Sun Yat-sen University Cancer Center, State Key Laboratory of Oncology in South China, Collaborative Innovation Center for Cancer Medicine, Guangzhou, China

${ }^{2}$ Department of Breast Cancer, Cancer Center, Guangdong Provincial People's Hospital, Guangdong Academy of Medical Sciences, Guangzhou, China

${ }^{3}$ Department of Pathology and Laboratory Medicine, Perelman School of Medicine, University of Pennsylvania, Philadelphia, PA, USA
}

\section{Corresponding Authors:}

Feng Ye and Xiaoming Xie, Department of Breast Oncology, Sun Yat-sen University Cancer Center, State Key Laboratory of Oncology in South China, Collaborative Innovation Center for Cancer Medicine, Guangzhou 510060, China.

Emails: yefeng@sysucc.org.cn; xiexm@sysucc.org.cn 
prognosis and short median survival. The median survival time of those patients without any treatment was about 4-8 months ${ }^{8}$ compared to 13-31 months ${ }^{2,3,9,10}$ after systemic treatment. For the treatment, systematic therapy is still the backbone to treat breast cancer with liver metastasis patients although surgery, radiofrequency ablation, or radiotherapy can be used for liver metastasis. ${ }^{11,12}$

Currently, the occurrence of liver metastasis from breast cancer cannot be accurately predicted. The construction of nomograms based on known prognostic factors is increasing and widely used to predict specific outcomes. ${ }^{13,14}$ We hypothesized a nomogram could be constructed by combining selected clinical and pathological variables using a multivariate model to predict the likelihood of postoperative liver metastasis in early breast cancer patients. This nomogram can be used to identify subgroups of high-risk patients, develop targeted screening and new preventive treatment strategies for early-stage breast cancer patients, and even improve life quality and survival outcomes. ${ }^{15,16}$ Therefore, we constructed and validated such a nomogram using retrospectively study data from 2 breast cancer patient populations.

\section{Methods}

The electronic database of the department of breast cancer at Sun Yat-sen University Cancer was searched and retrospectively reviewed from January 2008 to December 2010. Information from 1840 consecutive patients with nonmetastatic breast cancer was collected to serve as the basis for this study. The inclusion criteria for this study were: (1) female; (2) stage I-III breast cancer; (3) underwent mastectomy or breast-conserving surgery; (4) confirmed by the pathological diagnosis as invasive carcinoma. Exclusion criteria were: (1) male; (2) distant metastasis at initial diagnosis; (3) incomplete information such as TNM staging or pathological diagnosis; (4) other primary malignancies, including pre-diagnosis or follow-up of breast cancer. The staging of the tumor was based on the eighth edition of the TNM malignant tumor classification. The primary tumor and metastasis were confirmed by pathology. The classification of tumor size is: the maximum diameter of T1 tumor $\leq 20 \mathrm{~mm}$; the maximum diameter of T2 tumor is $>20 \mathrm{~mm}$, but $\leq 50 \mathrm{~mm}$; The maximum diameter of $\mathrm{T} 3$ tumor is $>50 \mathrm{~mm}$; $\mathrm{T} 4$ is directly invading the chest wall or skin regardless of tumor size. The diagnostic criteria for breast cancer liver metastasis were as follows: (1) BCLM was confirmed by histopathological examination;(2) When the patient is unable to undergo the pathological examination of liver metastases, we mainly diagnose BCLM based on clinical manifestations and imaging examinations. For patients without pathological examination, breast oncologists and imaging physicians jointly confirm the diagnosis of BCLM based on ultrasound, CT, and MRI.

Consecutive patients from January 2008 to December 2009 were included in the training cohort, while consecutive patients from January 2010 to December 2010 were included in the internal validation cohort from the same institutional database using the same criteria as the derived cohort.
The database includes the patient's treatment and pathological variables (age, menopausal status, tumor size, lymph node metastasis, histological grade, ER, PR, HER2 and lymphatic vessel invasion, etc.). The criteria for HER2 positivity is IHC $3+$ (defined as $>30 \%$ of invasive tumor cells with uniform strong membrane staining) or FISH amplification (defined as the ratio of HER2 to CEP17 > 2.2 or the average copy number of the HER 2 gene has a signal/core ratio greater than 6 when no internal reference probe is used in this detection system).

The retrospectively maintained database of early breast cancer patients, as well as this study, was approved by the Institutional Review Board of the Sun Yat-sen University Cancer Center.

\section{Statistical Analysis}

Statistical analysis was performed using SPSS ${ }^{\circledR}$ version 21.0 (IBM, Armonk, New York, USA) and statistical software package $\mathrm{R}$ version 3.5.1 (http://www.r-project). The clinical characteristics of the patients are mainly summarized as the variables of the classification. Comparison between groups was performed using a chi-square test to analyze categorical variables. Receiver Operating Characteristics (ROC) curve was built using SPSS ${ }^{\circledR}$ software. We firstly used univariate and multivariate analysis to determine factors that predict postoperative liver metastasis. Then, we identified the factors predicting postoperative liver metastasis by using a binary logistic regression model. Subsequently, we constructed a nomogram for predicting postoperative liver metastasis from the results of multivariate analysis using the rms package in $\mathrm{R}$. Internal verification of the nomogram was performed with bootstraps with 1000 resampling. Later, we evaluated the predicted performance of the established nomogram by $\mathrm{C}$-index measurement and the calibration curve is plotted to assess the calibration of the model, tested by Hosmer-Lemeshow test [20]. Decision curve analysis in the internal validation was set to determine the clinical value of the nomogram by quantifying the net benefit when considering different threshold probabilities [21, 22]. Besides, we constructed a clinical impact curve to assess the clinical impact of risk prediction models with decision curve analysis [23]. Finally, the breast cancer cohort of the SEER database was used for external validation.

\section{Results}

A total of 817 patients underwent breast-conserving surgery (including axillary lymph node dissection or sentinel lymph node biopsy); 1023 patients underwent modified radical mastectomy. The distribution of molecular subtypes in the training set is $68.8 \%$ in HR+/HER2-, $14.3 \%$ in HR+/HER2+, $7.0 \%$ in HR-/HER $2+$, and $10.0 \%$ in TN subtypes. The distribution of molecular subtypes in the verification concentration is: $63.5 \%$ in HR + /HER2-, $11.1 \%$ in HR + / HER2 +, 7.5\% in HR - / HER $2+$, and $12.0 \%$ in TN subtype. Our data analysis showed that in the training group of 1,149 patients with early breast 
Table I. Clinical and Pathological Features of Patients in the Training and Validation Cohort Based on Liver Metastatic Status.

\begin{tabular}{|c|c|c|c|c|c|c|}
\hline & \multicolumn{3}{|c|}{ Training cohort $(\mathrm{n}=1149)$} & \multicolumn{3}{|c|}{ Validation cohort $(n=691)$} \\
\hline Age, years & & & 0.811 & & & 0.187 \\
\hline$\leq 35$ & $4(3.6)$ & $108(96.4)$ & & $5(7.2)$ & $64(92.8)$ & \\
\hline$>35$ & $47(4.5)$ & $990(95.5)$ & & $23(3.7)$ & $599(96.3)$ & \\
\hline Negative & $19(5.6)$ & $321(94.4)$ & & 7 (3.6) & $189(96.4)$ & \\
\hline PR & & & 0.631 & & & \\
\hline Positive & $39(4.7)$ & 797 (95.3) & & $17(3.6)$ & $456(96.4)$ & 0.408 \\
\hline Negative & $12(3.8)$ & $301(96.2)$ & & II (5.0) & $207(95.0)$ & \\
\hline HER2 ${ }^{\circ}$ & & & 0.021 & & & 0.010 \\
\hline No & $48(4.3)$ & 1078 (95.7) & & $25(3.8)$ & $636(96.2)$ & \\
\hline Tumor size & & & $<0.001$ & & & $<0.001$ \\
\hline TI-2 & $34(3.3)$ & 994 (96.7) & & $19(3.0)$ & $621(97.0)$ & \\
\hline T3-4 & $17(14.0)$ & $104(86.0)$ & & $9(17.6)$ & $42(82.4)$ & \\
\hline lymph node metastasis & & & $<0.001$ & & & $<0.001$ \\
\hline Yes & $37(6.7)$ & $514(93.3)$ & & $26(7.7)$ & $313(92.3)$ & \\
\hline No & $14(2.3)$ & $584(97.7)$ & & $2(0.6)$ & $350(99.4)$ & \\
\hline Menopause at diagnosis & & & 0.881 & & & 0.174 \\
\hline Yes & $32(4.3)$ & $391(95.7)$ & & $8(2.8)$ & $280(97.2)$ & \\
\hline No & $19(4.6)$ & $707(95.4)$ & & $20(5.0)$ & $383(95.0)$ & \\
\hline Histological grade & & & 0.330 & & & 0.253 \\
\hline G1 or $G 2$ & $41(4.9)$ & $802(95.1)$ & & $19(3.6)$ & $513(96.4)$ & \\
\hline
\end{tabular}

cancer, 51 patients $(4.44 \%)$ had clinical evidence of liver metastasis after a median follow-up of 71 months. There are 4 patients with extensive systemic metastases, 2 patients with lung metastases, 2 patients with bone metastases, and 1 patient with brain metastases. The demographic and clinical characteristics of the patients in both cohorts are summarized in Table 1 . In the internal validation cohort, 28 patients $(4.05 \%)$ developed liver metastasis after a median follow-up of 59 months. There was no significant difference in liver metastasis between the 2 cohorts $(P=0.723)$. In univariate analysis, HER2 status, tumor size, and lymph node metastasis were associated with liver metastasis in breast cancer (Table 1). Subsequent liver metastasis was not significantly associated with age at diagnosis $(P=0.062)$, ER status $(P=0.271), \mathrm{PR}$ status $(P=0.361)$, lymphovascular invasion $(P=0.078)$, pathologic stage $(P=0.33)$ or menopause status at diagnosis $(P=0.881)$. In addition, we also used Cox regression to analyze the time-dependent risk factors for liver metastasis. The results of the training cohort suggested that HER2 status (Hazard ratio (HR) 1.80, 95\% CI (1.01-3.22); $\mathrm{P}=0.047)$, tumor size (HR 4.45, 95\% CI (2.41-8.24); $\mathrm{P}<0.001)$ and lymph Node metastasis (HR 2.19, 95\%CI (1.15-4.15); $\mathrm{P}=0.016)$ were independent risk factors for liver metastasis (Table. S1). The
COX regression of the internal validation cohort basically obtained similar results, although the effect of HER2 status on the recurrence time of liver metastasis did not reach statistical significance.

\section{Development of Nomogram}

Logistic regression model identified 3 variables associated with liver metastasis: tumor size (odds ratio (OR) 3.62, 95\% CI, 1.91 to $6.87 ; P<0.001$ ), lymph node metastasis (OR 2.26, 95\% CI, 1.18 to 4.34$) ; P=0.014$ ) and HER2 status (OR 1.86, 1.02 to $3.41 ; P=0.045$ ) (Table 2).

A nomogram containing these 3 factors was constructed (Figure 1). Good agreement between prediction and observation was displayed by the calibration curve of the training group (Figure S1A, supporting information). The Hosmer-Leme display test yielded a $\mathrm{P}$ value of 0.853 , indicating that the model fit well. The C-index of the predicted nomogram is 0.699 .

\section{Validation of Nomogram}

The calibration curve shows good agreement between predicted and observed liver metastasis in the internal validation set 
Table 2. Risk Factors for Liver Metastasis as Determined by Logistic Regression.

\begin{tabular}{|c|c|c|c|c|c|c|}
\hline Variables & \multicolumn{2}{|c|}{ Training cohort $(\mathrm{n}=1149)$} & \multicolumn{2}{|c|}{ Validation cohort $(n=691)$} & \multicolumn{2}{|c|}{ SEER Cohort $(n=265686)$} \\
\hline Tumor size & $3.62(1.91-6.87)$ & $<0.001$ & $4.53(|.87-| I .0)$ & $<0.001$ & I.88(I.74-2.04) & $<0.001$ \\
\hline Lymph node metastasis & $2.26(1.18-4.34)$ & 0.014 & I I.3(2.63-48.6) & $<0.001$ & $6.72(6.12-7.38)$ & $<0.001$ \\
\hline HER2 status & $\mathrm{I} .86(\mathrm{I} .02-3.4 \mathrm{I})$ & 0.045 & $2.36(1.04-5.35)$ & 0.04 & $3.11(2.87-3.37)$ & $<0.001$ \\
\hline C-index & 0.699 & & 0.814 & & 0.791 & \\
\hline
\end{tabular}

OR: Odds Ratio.

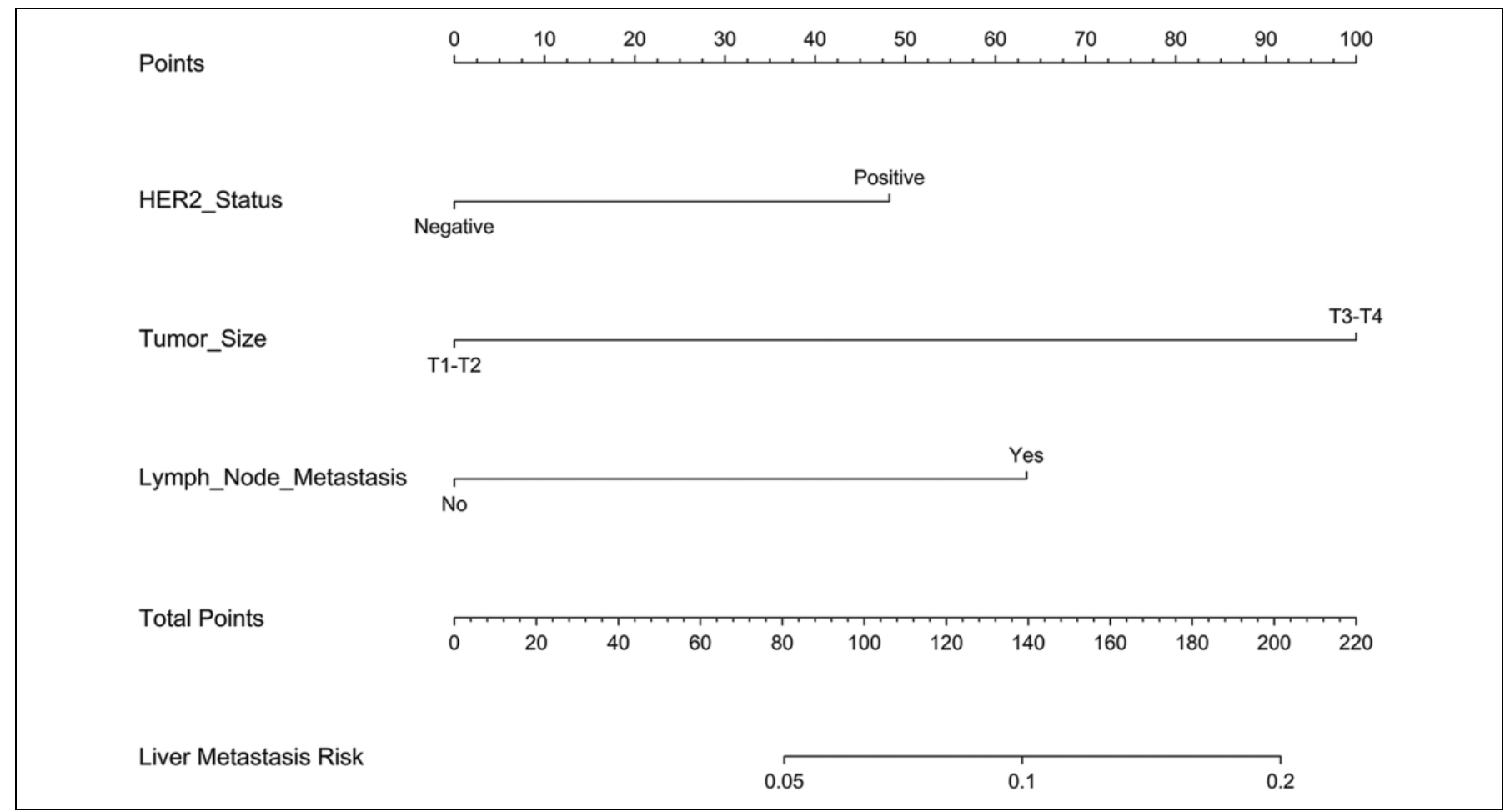

Figure I. Nomogram to predict postoperative liver metastasis in patients with nonmetastatic breast cancer. There are 6 rows in the nomogram. Significant variables are displayed in lines 2 through 4, and the points of each variable are read from the scale of line I. Add the points of the 3 variables to the total and mark them on the scale of line 5 . The risk of liver metastasis is read from the scale of line 6 by drawing a vertical line from the total point marked in line 5 and the points are translated to the probability of liver metastasis.

(Figure S1, supporting information) due to the non-significant $\mathrm{P}$ value $(0.972)$ produced by the Hosmer-Lemeshow test. The C-index of the predicted nomogram is 0.814 . The ROC curve was constructed for the derived and validated groups (Figure 2). For the training and verification groups, the area under the curve (AUC) is 0.699 and 0.815 , respectively, and the corresponding cutoff values are 0.052 and 0.031 .

Furthermore, we used the breast cancer cohort of the SEER database to further validate this nomogram. The results of the SEER database further confirmed the results of the training cohort, and also supported that HER2 status (odd ratio (OR) 1.88 , 95\%CI (1.74-2.04); $\mathrm{P}<0.001)$, tumor size (OR 6.72, $95 \%$ CI $(6.12-7.38) ; \mathrm{P}<0.001)$ and lymph node metastasis(OR $3.11,95 \% \mathrm{CI}(2.87-3.37) ; \mathrm{P}<0.001)$ were risk factors for breast cancer liver metastasis. The C-index of the SEER cohort nomogram is 0.791 . The calibration curve showed good agreement between predicted and observed liver metastasis in the SEER cohort.

\section{Clinical Use}

The decision curve analysis of the nomogram is shown in Figure 3A. This analysis showed that when the threshold probability was in the range of $0-0.7$, the use of a nomogram to predict liver metastasis increased more net benefit than the treat-all or treat-none strategy. Within this range, the net benefit based on the nomogram overlaped at several points. 

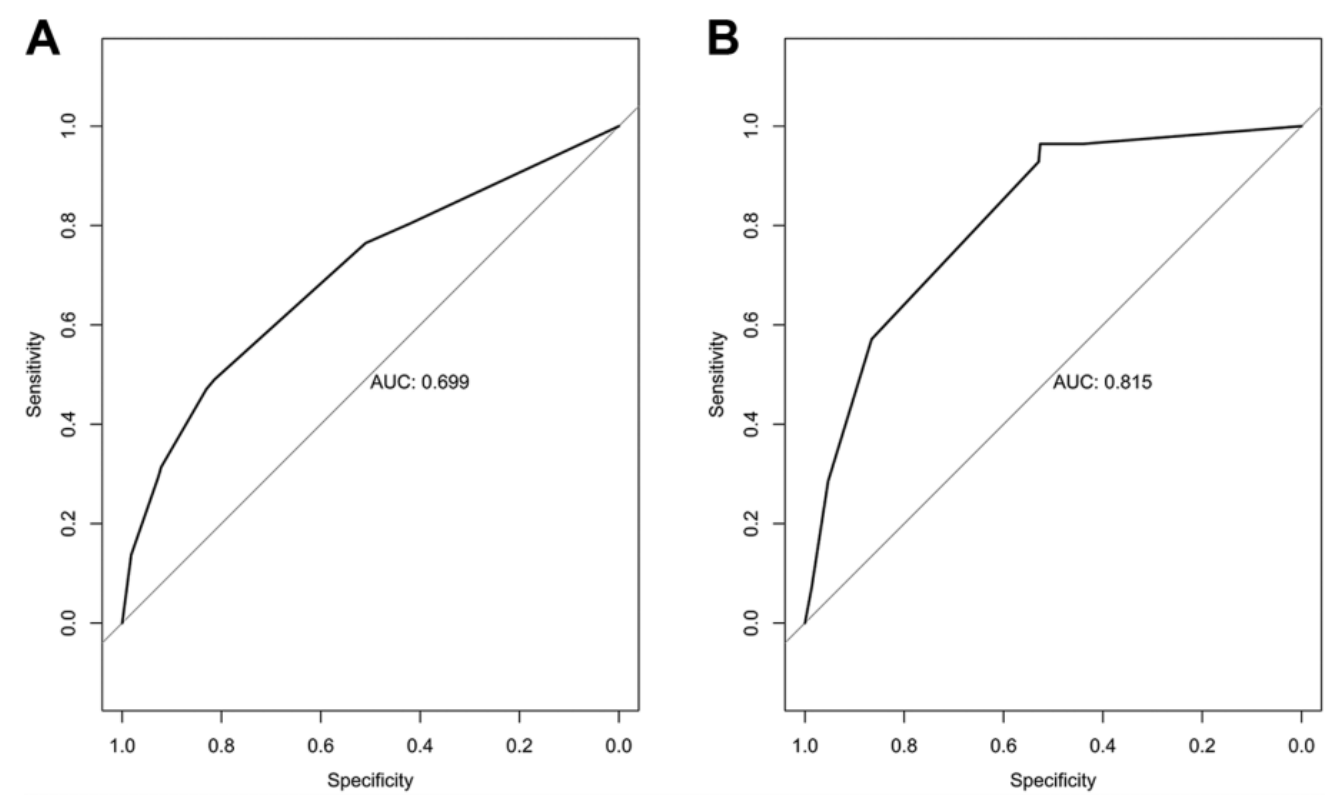

Figure 2. The Receiver operating characteristic (ROC) curves for training $(A)$, internal validation (B) cohorts; Areas under the ROC curve are $0.699(\mathrm{~A})$ and $0.8 \mathrm{I} 5$ (B). Cut-off values (marked with a symbol) are $0.052(\mathrm{~A})$ and $0.03 \mathrm{I}(\mathrm{B})$.

The clinical impact curve analysis of the nomogram was shown in Figure 3B. The red curve (Number high risk) indicated the number of people who were classified as positive (high risk) by the simple model at each threshold probability; the blue curve (Number high risk with events) was the number of true positive at each threshold probability.

\section{Discussion}

We have developed and validated a nomogram that predicts the development of postoperative liver metastasis in early breast cancer patients. The nomogram included 3 items, tumor size, lymph node metastasis, and HER2 status, and showed good agreement between the predicted and actual probabilities in the derived and validated cohorts.

Liver metastasis is a growing problem in the treatment of breast cancer. ${ }^{17}$ Liver metastasis severely affects patients' life quality and prognosis. Therefore, predicting higher risk liver metastasis breast cancer patients will enrich the population who should be treated more specifically and thereby improve clinical outcomes in these patients. ${ }^{18}$

Based on this nomogram, assuming a breast cancer patient with T3-4, lymph node metastasis and HER2-positive tumors, her total score was 205, as shown in Figure 1. Using a nomogram, the patient is expected to have a $20 \%$ possibility to develop liver metastasis. Therefore, patients with the above characteristics are expected to benefit from liver metastasis screening.

In contrast, an assuming patient with a T1-2 tumor, no lymph node metastasis, and the HER2 negative status had a total score of 0, as shown in Figure 1. Using a nomogram, the predicted chance for this patient to get liver metastasis is relatively low (less than 5\%).

There is currently no specific preventive treatment to reduce the incidence of liver metastasis in breast cancer. But due to the local liver treatment (surgery, intrahepatic local chemotherapy, etc.), strengthen surveillance may bring benefits for high-risk metastatic breast cancer patients. We are not the only ones trying to establish a nomogram on breast cancer liver metastasis. Lin and his colleagues constructed a nomogram using variables such as sex, histology type, $\mathrm{N}$ stage, grade, age, ER, PR, HER2 status. ${ }^{19}$ The problem with their nomogram is that the patients they enrolled are de novo liver metastasis, which means that the diagnosis of liver metastasis and the diagnosis of breast cancer are simultaneous and thus it doesn't have enough predictive value. The patients included in this study were those who had liver recurrences after early breast cancer treatment. Thus, our nomogram has a more superior predictive value than theirs. Additionally, when $\mathrm{T} 1$ and $\mathrm{T} 2$ are divided into 2 groups, the ROC curve and the calibration curve of this new nomogram are almost the same as the previous results (Figure S2, supporting information).

It is worth noting that this study also has some limitations. First of all, this nomogram was constructed using retrospective data, so prospective studies should be performed for further validation. Secondly, we did not evaluate the impact of adjuvant $\mathrm{CT}$, endocrine, and targeted therapy due to the unavailability of data. Thirdly, we also did not evaluate the effect of the eighth edition of TNM staging on liver metastasis due to the unavailability of data. Finally, the AUC in the ROC analysis of the training cohort is relatively low. 

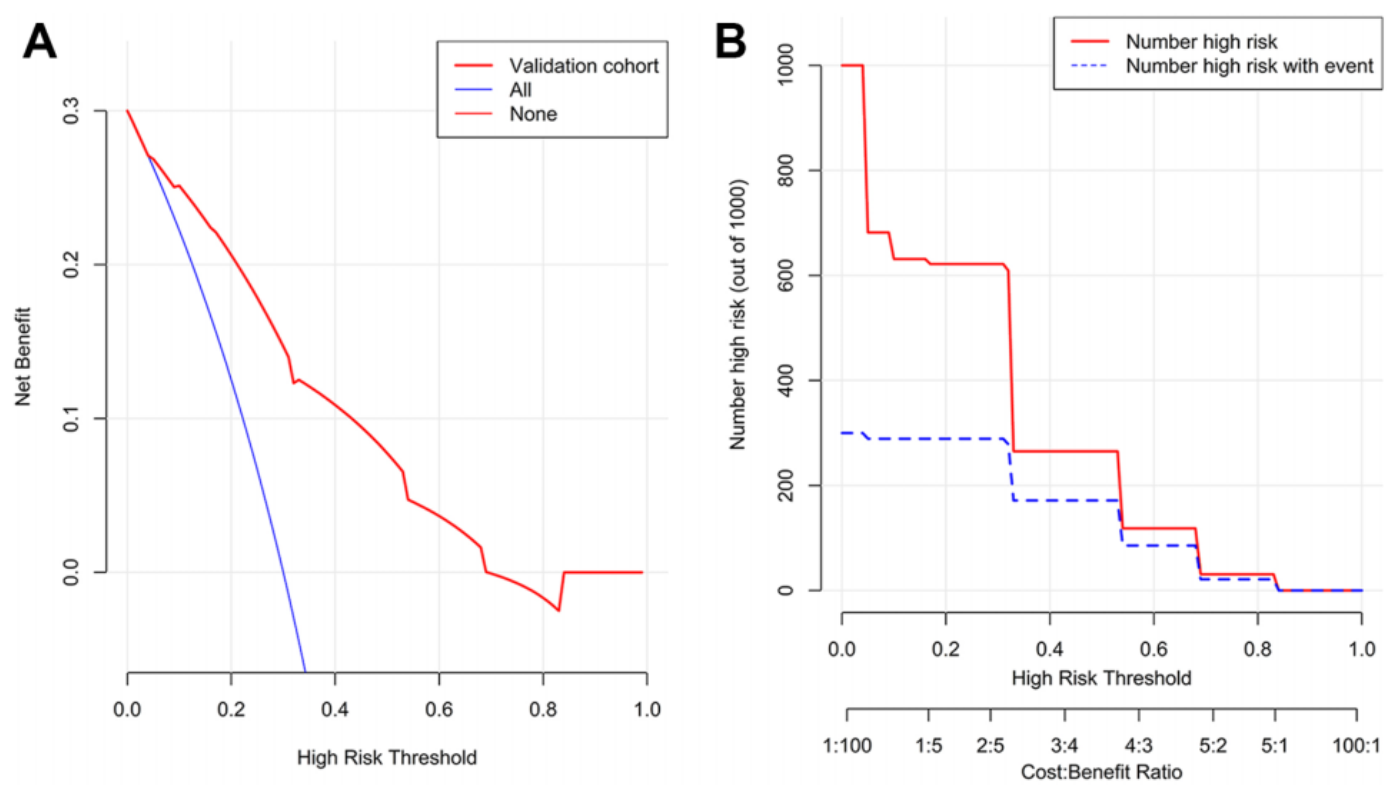

Figure 3. Decision curve analysis $(A)$ and clinical impact curve analysis $(B)$ of the validation cohort. $(A)$ : The $y$-axis represents net benefit. The $\mathrm{x}$-axis shows the threshold probability. "All" means that all patients have assumed liver metastasis, and "none" means that the patient has no liver metastasis. Using a nomogram to predict, if the score is in the range of 0 to 0.7 , liver metastasis increases more net benefit than the treat-all or treat-none strategy. (B): The red curve (Number high risk) indicates the number of patients who are classified as positive (high risk) by the simple model at each threshold probability; the blue curve (Number high risk with events) is the number of true positives at each threshold probability.

\section{Conclusion}

In summary, we developed a nomogram, which is a powerful tool for predicting subsequent liver metastasis in nonmetastatic breast cancer patients. Our model will help us in identifying patients at high risks of liver metastasis, thereby we could design preventive trials for them correspondingly. Further researches are needed to determine whether it can be applied to other subgroups of patients.

\section{Acknowledgments}

We thank the staff in the Medical Records Management Section of the Sun Yat-sen University Cancer Center for supporting the research.

\section{Author Contribution}

Anli Yang, Weikai Xiao, and Shaoquan Zheng contributed equally to this study.

\section{Availability of Data and Materials}

Data supporting the results of this study can be obtained from the corresponding author under reasonable request.

\section{Declaration of Conflicting Interests}

The author(s) declared no potential conflicts of interest with respect to the research, authorship, and/or publication of this article.

\section{Funding}

The author(s) received no financial support for the research, authorship, and/or publication of this article.

\section{ORCID iD}

Anli Yang (D) https://orcid.org/0000-0003-0966-7285

\section{Ethics Statement}

This study was deemed exempt from institutional review board approval by Sun Yat-sen University Cancer Center and the informed consent was waived. This study was conducted in accordance with the ethical standards of the World Medical Association Declaration of Helsinki.

\section{Informed Consent}

Informed consent was obtained from all individual participants included in the study.

\section{Supplemental Material}

Supplemental material for this article is available online.

\section{References}

1. Siegel RL, Miller KD, Jemal A. Cancer statistics, 2018. CA Cancer J Clin. 2018;68(1):7-30.

2. Lobbezoo DJ, van Kampen RJ, Voogd AC, et al. Prognosis of metastatic breast cancer: are there differences between patients with de novo and recurrent metastatic breast cancer. Br J Cancer. 2015;112(9):1445-1451.

3. Ruiz A, van Hillegersberg R, Siesling S, et al. Surgical resection versus systemic therapy for breast cancer liver metastases: results of a European case matched comparison. Eur J Cancer. 2018; 95:1-10 
4. Kennecke H, Yerushalmi R, Woods R, et al. Metastatic behavior of breast cancer subtypes. J Clin Oncol. 2010;28(20):3271-3277.

5. Duan XF, Dong NN, Zhang T, Li Q. The prognostic analysis of clinical breast cancer subtypes among patients with liver metastases from breast cancer. Int J Clin Oncol. 2013;18(1):26-32.

6. Illing R, Gillams A. Radiofrequency ablation in the treatment of breast cancer liver metastases. Clin Oncol (R Coll Radiol). 2010; 22(9):781-784.

7. Pentheroudakis G, Fountzilas G, Bafaloukos D, et al. Metastatic breast cancer with liver metastases: a registry analysis of clinicopathologic, management and outcome characteristics of 500 women. Breast Cancer Res Treat. 2006;97(3):237-244.

8. Adam R, Aloia T, Krissat J, et al. Is liver resection justified for patients with hepatic metastases from breast cancer. Ann Surg. 2006;244(6):897-907; discussion 907-908.

9. Ge QD, Lv N, Kong YN, et al. Clinical characteristics and survival analysis of breast cancer molecular subtypes with hepatic metastases. Asian Pac J Cancer Prev. 2012;13(10):5081-5086.

10. Leone BA, Vallejo CT, Romero AO, et al. Prognostic impact of metastatic pattern in stage IV breast cancer at initial diagnosis. Breast Cancer Res Treat. 2017;161(3):537-548.

11. Ruiz A, Wicherts DA, Sebagh M, et al. Predictive profile-nomogram for liver resection for breast cancer metastases: an aggressive approach with promising results. Ann Surg Oncol. 2017;24(2): 535-545.
12. Taşçi Y, Aksoy E, Taşkın HE, et al. A comparison of laparoscopic radiofrequency ablation versus systemic therapy alone in the treatment of breast cancer metastasis to the liver. HPB (Oxford). 2013;15(10):789-793.

13. Lerner SP, Bochner B, Kibel AS. The use and abuse of data: nomograms and talking to patients about clinical medicine. Urol Oncol. 2007;25(4):333-337.

14. Xie X, Tan W, Chen B, et al. Preoperative prediction nomogram based on primary tumor miRNAs signature and clinical-related features for axillary lymph node metastasis in early-stage invasive breast cancer. Int J Cancer. 2018;142(9):1901-1910.

15. Graesslin O, Abdulkarim BS, Coutant C, et al. Nomogram to predict subsequent brain metastasis in patients with metastatic breast cancer. J Clin Oncol. 2010;28(12):2032-2037.

16. Zheng $\mathrm{H}$, Tang $\mathrm{H}$, Wang $\mathrm{H}$, et al. Nomogram to predict lymph node metastasis in patients with early oesophageal squamous cell carcinoma. Br J Surg. 2018;105(11):1464-1470.

17. Ma R, Feng Y, Lin S, et al. Mechanisms involved in breast cancer liver metastasis. J Transl Med. 2015;13:64.

18. Golubnitschaja O, Sridhar KC. Liver metastatic disease: new concepts and biomarker panels to improve individual outcomes. Clin Exp Metastasis. 2016;33(8):743-755.

19. Lin Z, Yan S, Zhang J, Pan Q. A Nomogram for distinction and potential prediction of liver metastasis in breast cancer patients. J Cancer. 2018;9(12):2098-2106. 